\title{
EL CARÁCTER ANTIDEMOCRÁTICO DE LA CONSTITUCIÓN DE 1917: SU RECEPCIÓN EN LAS ENTIDADES FEDERATIVAS, PARTICULARMENTE EN EL ESTADO DE AGUASCALIENTES
}

\author{
The Antidemocratic Character of the Constitution of 1917: \\ its Reception in Federal Entities, Specially in the State of Aguascalientes \\ José Alfredo MUÑOZ DELGADO*
}

\begin{abstract}
Sumario:
I. Introducción II. Antecedentes III. La revolución constitucionalista IV. La Constitución de 1917: de origen espurio a legitimación posterior V. La recepción de la Constitución mexicana de 1917 por los Estados de la República VI. El constitucionalismo en Aguascalientes producto de la Constitución mexicana de 1917 VII. Reflexión final VIII. Fuentes bibliográficas
\end{abstract}

Resumen: La Constitución de 1917, producto de la propuesta de Venustiano Carranza, es una Constitución impuesta por las armas y no por los votos, decisión de una minoría y por ende no democrática, que obligó a los Estados de la República a la armonización de sus constituciones locales con la Federal, obteniendo poco a poco su aceptación. En el Estado Mexicano la simulación constitucional ha definido al Derecho público, ocasionando un sentimiento de comodidad y preocupación por conservarla.

Palabras Claves: Constitución, legitimación, democracia, revolución.

Abstract: The Constitution of 1917, product of the proposal of Venustiano Carranza, is a Constitution imposed by arms and not by votes, decision of a minority and therefore undemocratic, which forced the States of the Republic to harmonize their constitutions local with the Federal, getting its acceptance little by little. In the Mexican State, constitutional simulation has defined public law, causing a feeling of comfort and concern to preserve it.

Keywords: Constitution, legitimacy, democracy, revolution.

\section{Introducción}

Entre las muchas entregas periódicas de José Vasconcelos, encontramos, una a la que quiero referirme, ¿Qué es la Revolución?' La define, como el recurso colectivo de las armas para derribar

\footnotetext{
* Doctor en Derecho por la Universidad Michoacana de San Nicolás de Hidalgo. Profesor investigador de la Universidad Autónoma de Aguascalientes. Miembro del claustro docente de la Maestría y del Doctorado Interinstitucional en Derechos Humanos en el que participan la Comisión Nacional de Derechos Humanos y las universidades: Autónoma de Baja California Sur, Autónoma de Campeche, de Guanajuato y Autónoma de Tlaxcala.

1 VAsconcelos, José (2009), ¿Qué es la Revolución?, Editorial Trillas, Biblioteca José Vasconcelos No. 7, México, pp. 47-50. 
opresiones ilegítimas y reconstruir la sociedad sobre bases de economía sana y moral elevada. La justifica porque es el medio para crear un Estado Social más justo y más libre que el régimen que se ha destruido o intenta destruir. Concluye, toda revolución que lo es de verdad combate y destruye, pero sólo mientras está en las barricadas, cuando se constituye en gobierno tiene que volverse creadora y serena, constructiva y justa. De alguna manera es esta la historia de una de las etapas de la Revolución Mexicana de 1917, la Constitucionalista, de la revolución carrancista que para 1916 ha derrotado a sus opositores, a Francisco Villa en Celaya, Estado de Guanajuato y a Emiliano Zapata reconcentrándolo al Estado de Morelos, pudiendo gobernar, así sea transitoria o provisionalmente, pero sin dificultad.

Las revoluciones devoran a sus hijos, por eso la prisa en institucionalizarlas, porque la revolución permanente se convierte en desorganización y decadencia y el último en sobrevivir se quedará para siempre en el poder, convirtiéndose en el opresor que derrocó, lo que no se evita ni con una nueva constitución.

\section{Antecedentes}

El movimiento armado que inicia Francisco I. Madero en 1910 es el vendaval que desata en México no una sino varias y diversas revoluciones. Venustiano Carranza hace la suya, la Constitucionalista, no porque de entrada quisiera una nueva constitución, sino porque exigía se cumpliera la existente, la de 1857 .

Con la bandera de la legalidad, el 19 de febrero de 1913, el Congreso del Estado de Coahuila de Zaragoza emitió el decreto número $1421^{2}$, con apenas dos artículos y una exhortación, en el primero se desconoce a Victoriano Huerta como Jefe del Poder Ejecutivo de la República y todos los actos y disposiciones que dicte con ese carácter; en el segundo se conceden facultades extraordinarias a Carranza para que proceda a armar fuerzas para coadyuvar en el sostenimiento del orden constitucional de la República. Finalmente en un artículo económico, se exhorta a los gobiernos de los estados y jefes de las fuerzas federales, rurales y auxiliares para que secunden la actitud del Gobierno del Estado de Coahuila.

Este y muchos otros decretos se publicaban en El Constitucionalista órgano oficial de la Primera Jefatura, editado en Hermosillo, Sonora.

Cuando Carranza conoció el mensaje que el General Victoriano Huerta envió a los gobiernos locales, comunicándoles que con autorización del senado de la República se había hecho cargo del poder ejecutivo federal, estando presos el presidente de la República y todo su gabinete en una circular ${ }^{3}$, se apresuró a desconocerlo y exhortó a desplegar la bandera de la legalidad para sostener al gobierno constitucional emanado de las últimas elecciones de 1910 y en todo caso respetar la Constitución convocando a elecciones extraordinarias.

Acusando de traición a Huerta, Carranza, el 26 de marzo de 1913, con sus jefes y oficiales con mando de fuerzas constitucionalistas, manifiesta sostener con las armas el Plan de Guadalupe, llamado así por haberse firmado en la hacienda de Guadalupe, Coahuila, que fuera secundado

\footnotetext{
2 Gobierno Provisional de la República Mexicana (1915), Codificación de Decretos del C. Venustiano Carranza, Primer Jefe del Ejército Constitucionalista, encargado del Poder Ejecutivo de la Unión, Imprenta de la Secretaria de Gobernación, México, pp. 5 y 6.

3 "Circular en la que se excita al movimiento legitimista", El Constitucionalista, No. 3, Hermosillo Sonora, 6 de diciembre de 1913 .
} 
por otros jefes de armas y desde luego con formalidad por el Gobierno del Estado de Coahuila, cuya Diputación Permanente emite el decreto 1498, adhiriéndose, el 19 de abril de 1913.

Esto revela una actitud legitimadora, donde se advierte la obsesión legalista de Carranza, que en su mejor momento lo llevará a proponer una reforma revolucionaria de la constitución de 1857 sin importarle forzar los procedimientos e incumplir con la legalidad que decía defender, derribando él mismo el árbol a cuya sombra se acogía, porque no pocas veces le dificultaba o de plano no le permitía realizar sus proyectos.

Habría que hacerse una pregunta: ¿tenía el Congreso del Estado de Coahuila facultades constitucionales para conferir el derecho a la revolución, que en realidad significa el desconocimiento de la legalidad? Esta paradoja nos permite entender que la legalidad carrancista era sólo un pretexto.

En la circular ya referida en la que se excita al movimiento legitimista ${ }^{4}$, asevera que la designación que el Senado hace de Victoriano Huerta como Presidente de la República es arbitraria e ilegal, acusa al Senado de connivencia y complicidad con los malos soldados, enemigos de nuestra patria y libertades.

En el ascenso de Victoriano Huerta al poder, afirma Tena Ramírez ${ }^{5}$, se habían observado impecablemente las formalidades constitucionales, ocurriendo todo en apenas unas horas del día 19 de febrero de 1913 .

En un primer decreto presentado ante la Cámara de Diputados, con la dispensa de trámites, se aprueban de tres puntos resolutivos, relativos a las respectivas renuncias, tanto del presidente Madero, a quien le fue aceptada con la aprobación 123 votos a favor y sólo 5 en contra, cuanto al vicepresidente Pino Suarez, con 119 a favor y 8 en contra. En el tercer resolutivo de los aprobados se llama al ciudadano Pedro Lascuráin, Secretario del Despacho de Relaciones Exteriores para que rindiera protesta como presidente interino de la República, aprobándolo en votación económica.

A continuación en una sesión extraordinaria del congreso general, celebrada ya por la noche, Lascuráin rinde su protesta como presidente interino, para luego en una sesión extraordinaria de la Cámara de Diputados se les hiciera saber que el presidente interino, Lascuráin, había nombrado con esa esa misma fecha - apenas unos momentos antes- al General de División, Victoriano Huerta, como Secretario de Estado y del Despacho de Gobernación presentando acto continuo la renuncia del Presidente de la Republica, la cual se aprueba en votación económica, por lo que, admitida la renuncia, se llama al ciudadano Victoriano Huerta para que rinda protesta de ley como presidente interino. En la misma sesión extraordinaria del congreso general, Huerta rinde protesta como Presidente Interino de los Estados Unidos Mexicanos.

Así en unas pocas horas de ese día, Huerta había legitimado constitucionalmente su ascenso al poder. Lo que no cuenta Tena Ramírez es que el puente constitucional fue obra de su admirado maestro, Emilio Rabasa, entonces senador por el Estado de Chiapas, combinado con la connivencia del propio Madero y Pino Suárez, quien en una carta ${ }^{6}$ dirigida a su amigo Serapio Rendón, escrita mientras estaban presos en la intendencia de Palacio Nacional, le hace saber que fueron obligados a renunciar a sus cargos, y aun sabiendo que sus vidas no estaban a salvo,

\footnotetext{
4 Ibídem.

5 Tena Ramírez, Felipe (1981), Derecho Constitucional Mexicano. Editorial Porrúa. México, p. 69.

6 Solares, Ignacio (2009), Ficciones de la Revolución Mexicana. Alfaguara, México, p. 11.
} 
no tuvieron la entereza de negarse, con la esperanza de salvar la vida a costa de la honra, empequeñeciéndose.

Debo decir en defensa de Madero, tomando los calificativos que de él hace Ignacio Solares ${ }^{7}$, que era un buen hombre, que no fusilaba a nadie, hiciera lo que hiciera. No lo hizo cuando su hermano Gustavo irrumpió en su despacho, colocándole una pistola a Huerta en la sien, luego de haber descubierto los planes del cuartelazo. Pero Madero le creyó a Huerta, condenando a una horrible muerte a su hermano. Era un hombre de gran sencillez y candor, que quería gobernar con y para la libertad, no estaba hecho para la barbarie sangrienta de nuestra revolución.

La debilidad de Madero, causa del odio que por él sentía Bernardo Reyes, quien decía que era una debilidad sucia ${ }^{8}$, y la habilidad de Rabasa, hicieron que Victoriano Huerta, un chacal, sin duda, pero no un usurpador, con una combinación de actores y circunstancias legitimara constitucionalmente su ascenso a la Presidencia de la República.

Emilio Rabasa, un porfiriano eminente y el jurista que más influencia tuvo en la Constitución de 1917, fue el artífice. Hay un hecho que lo revela y que cuenta Carlos Monsiváis9.

En 1913, al cabo del lamentable suceso de la Ciudadela conocido como la Decena Trágica, Emilio Rabasa, encabezando un grupo de senadores, le pide la renuncia al Presidente Madero, quien lacónicamente les responde: No me llama la atención que ustedes vengan a exigirme la renuncia, porque los senadores nombrados por el general Díaz y no electos por el pueblo, me consideran enemigo y verían con gusto mi caída.

La renuncia de Madero vendría cuando la exige Huerta acompañada de la amenaza de muerte, pues era crucial para sostener el puente constitucional, por eso la insistencia. Por esto, Rabasa consigue que Huerta le proponga la Rectoría de la Universidad Nacional y, aunque no la acepta, unen sus destinos. A la caída de Huerta, Rabasa se exilia en Nueva York.

Las cuestiones de legalidad se le dieron mejor a Huerta que a Carranza. Aquél, contando con Rabasa tendría la ley en sus manos; éste tuvo que forzar los argumentos en pro de su bandera, empeñado en justificarse constitucionalmente sin lograrlo. Sólo la fuerza de sus armas terminaría haciéndolo, sólo con ellas podía garantizar el orden o, mejor dicho, sólo con ellas podía imponerlo.

Puente entre siglos, así describe Krauze a Carranza en su Biografía del Poder ${ }^{10}$, y lo era sin duda, formado en el siglo XIX, arriba al siglo XX, con el bagaje y los saberes de aquél, para continuar aplicándolos en las soluciones que necesitaba en éste, sólo así se explica la insistencia legalista que no consigue, pero de la que no abdica. Sería imposible saber si Carranza estaba consciente de que su pretendida legalidad era aparente, o si creía que la cumplía a pie juntillas, lo cierto es que en la historia de nuestro país es una constante enarbolar la ley y hacer otra cosa muy distinta de lo que esta exige.

Con los sucesos importantes que presencia el joven Carranza durante su estancia en la Ciudad de México - la caída de Sebastián Lerdo de Tejada y el ascenso de Díaz al poder con la Revolución de Tuxtepec- aprenderá la lección de la legalidad política a la mexicana, no siempre ajustada al derecho, pero muy útil para justificar y legitimar sus acciones, y de la que hará uso ya en el poder.

\footnotetext{
7 Solares, Ignacio (2013), Un sueño de Bernardo Reyes, Alfaguara, México, p. 10.

8 Ibidem.p. 12.

9 Monsiváis, Carlos (1986), Prólogo a La Bola de Emilio Rabasa, ediciones Océano, México, p. VII.

${ }^{10}$ Krauze, Enrique (1987), Venustiano Carranza. Biografía del Poder 5., Fondo de Cultura Económica, México. 


\section{La Revolución Constitucionalista}

Para entender esta cuestión es necesario revisar, así sea someramente, el episodio político jurídico que explica a mi modo de ver porqué se trata de una revolución constitucionalista y la bandera de la legalidad.

En nuestra historia constitucional se imbrican los aspectos políticos más importantes Un episodio de notable relevancia lo constituye el debate que sostuvieron José María Iglesias e Ignacio Luis Vallarta entre 1874 y 1881.

La cuestión de inicio: ¿Tiene la Suprema Corte de Justicia de la Nación competencia para resolver controversias sobre la legitimidad de un poder o de alguna autoridad? Las respuestas pueden ser puramente jurídicas, pero también y sobre todo políticas, si quien tiene que darlas está interesado en el poder de manera personal o en algún partido.

José María Iglesias, desde la Suprema Corte de Justicia de la Nación, da una respuesta afirmativa, y desarrolla su doctrina que conocemos como Incompetencia de Origen ${ }^{11}$, mediante la cual a través del juicio de amparo se cuestiona la falta de legitimidad de cualquier autoridad por vicios de legalidad, con independencia de su validación por el poder legislativo, que expuso a detalle con motivo del célebre caso conocido como Amparo Morelos, llamado así porque fue la Legislatura del Estado de Morelos que, en 1875, se opuso a que la corte decidiera sobre la legitimidad de los poderes locales. Vallarta en un principio estaba de acuerdo, aunque más tarde se opuso señalando que la Constitución - la de 1857- no concedía esta atribución a la corte, desde cuya presidencia, logra derribar la tesis de la incompetencia de origen.

Sin embrago esto no es cuestión de argumentos jurídicos, es cuestión de política resuelta con legalidad aparente, en busca de legitimación constitucional, protagonizada por los juristas más brillantes del siglo XIX mexicano. La agudeza de Carranza sin duda le permitió nutrirse de ello.

Recordemos que fue Ignacio Comonfort quien siendo presidente de la república se negó a jurar la constitución de 1857, pues con ella no se podía gobernar. Así lo justifica históricamente Emilio Rabasa en la Constitución y la Dictadura ${ }^{12}$, lo que le vale ser llamado, teórico de la dictadura necesaria ${ }^{13}$. Benito Juárez interpreta que se trata de un golpe de Estado, y él como Presidente de la Suprema Corte de Justicia de la Nación y Vicepresidente de la República se presenta en Palacio Nacional a ejercer la Presidencia conforme a las reglas constitucionales por ausencia del titular, según artículos 79 y 82 de la propia Carta Magna.

Una vez restaurada la República en 1867, Juárez derrota en las elecciones convocadas a Porfirio Díaz, héroe militar de la restauración. Para el siguiente periodo constitucional, en 1871, se encuentran nuevamente Juárez y Díaz, pero esta vez también contiende Sebastián Lerdo de Tejada, otrora ministro del gobierno juarista. La muerte del Presidente Juárez deja a Lerdo como presidente interino. Dos años más tarde en un hecho inusitado, Díaz, Vicente Riva Palacio y José María Iglesias, se inscriben para contender por la Presidencia de la Suprema Corte de Justicia de la Nación; en aquellos años era el cargo de elección popular y no se requería ser

\footnotetext{
${ }^{11}$ Iglesias, José María (1874), Estudio Constitucional Sobre las Facultades de la Corte Suprema, imprenta de Díaz de León y White, México.

${ }^{12}$ Rabasa, Emilio (1912), La Constitución y la Dictadura, tipografía de Revista de Revistas, México.

${ }^{13}$ Díaz y DíAz, Martín (1991), Emilio Rabasa: Teórico de la Dictadura Necesaria, Miguel Ángel Porrúa, México.
} 
jurista para ocuparlo, y era una gran puerta para ingresar a la Presidencia de la República, así lo entendió Porfirio Díaz.

Concluido el periodo presidencial de Sebastián Lerdo de Tejada, en 1876, Porfirio Díaz no se arriesgaría a perder una vez más, y para impedir la reelección se levanta en armas, proclamando el Plan de Tuxtepec con la bandera irónica, si de Díaz se trata, de la No Reelección, y depositando provisionalmente la presidencia de la república en el presidente de la suprema corte, en su defecto en el jefe de armas, o sea él. El artífice de esta idea no fue otro que Ignacio L. Vallarta.

Mientras inicia la revuelta, Lerdo es reelecto en 1876, pero es cuestionado por José María Iglesias, Presidente de la suprema corte, que desconoce la validez que de la elección había hecho el Congreso, y a la manera de Juárez, interpreta esto como un golpe de Estado. Así que con la connivencia de algunos estados, se proclama Presidente de la república. Díaz lo busca y trata de pactar a cambio de que le deje nombrar a los mandos militares, lo que Iglesias no acepta, pero con el triunfo en la batalla de Tecoac, Díaz asciende al poder y es legitimado en las elecciones de 1877 , en las que también se elige como presidente de la suprema corte a Ignacio L. Vallarta, quien tendrá la misión y el encargo de Porfirio Díaz, de reformar la constitución para que el presidente de la suprema corte no sustituyera en su ausencia al presidente de la república. Lo que logró con relativa facilidad empezando a desarrollar la doctrina constitucional que quitará a la Suprema Corte de Justicia de la Nación la facultad de resolver sobre la ilegitimidad del poder, acabando con el fantasma que perseguía a Porfirio Díaz comprometiendo su legitimidad. Así por más de un siglo y sin argumentos sólidos, sólo de autoridad, fue hasta 1995 cuando se creó la jurisdicción electoral a cargo del poder judicial de la Federación, como materia especializada, para solucionar la cuestión, a fuerza de no reconocer que José María Iglesias tenía la razón jurídica, pero quizá no la razón política.

La batalla por el poder se desarrolló en sede institucional, desde el Estado mismo. Salvo la revuelta de Tuxtepec, la disputa por el poder durante la vigencia de la constitución de 1857 tenía cauces legales y se construyeron muchas derivaciones. Durante la Revolución de 1910, Carranza construiría sus propios cauces legales. Artificiosamente se iría legitimando constitucionalmente, imponiéndose no con votos, sino con las armas.

La Constitución de 1857 no permitía solucionar un conflicto de legitimidad del poder, no existían los procesos constitucionales para ese tipo de controversias, así entonces Carranza hubo de encontrar la manera de hacer política aparentando legitimidad constitucional.

Una vez impuesto el orden por los constitucionalistas, Carranza, contando con la figura trascendental de Luis Cabrera, emprende el proyecto de realizar un cambio constitucional. Incorporando las banderas de sus opositores, más no a ellos, logra instaurar un Congreso Constituyente para el caso. Pero una vez más, la Constitución de 1857 sólo estaba en su discurso y desde hacía mucho tiempo no regía en México, y en esta ocasión tampoco.

Una reforma constitucional requería de realizarse mediante el procedimiento legislativo previsto en el artículo 127 de la Constitución de $1857^{14}$, que permite desde luego la reforma o la adición constitucional, encargándola a un poder revisor conformado por el Congreso de la Unión y las Legislaturas locales, exigiendo votaciones calificadas.

Venustiano Carranza se asumía como constitucionalista, pero transgredía la constitución, le estorbaba incluso, no la respetaba, la cláusula de inviolabilidad constitucional contenida en el artículo 128 simplemente no fue obstáculo alguno, una melodía que nadie atendió.

\footnotetext{
14 Texto Íntegro en Tena Ramírez, Felipe (1957), Leyes Fundamentales de México, Porrúa, México, pp. $595-629$. 


\section{La Constitución de 1917: de Origen Espurio a Legitimación Posterior}

¿Qué validez puede tener esta Constitución -1917- emanada de la violación de la anterior?, se pregunta Tena Ramírez ${ }^{15}$. Algunas respuestas: la constitución de 1857 no regía más en México pero era especialmente útil en el discurso constitucionalista de Carranza. Por ello propone una reforma revolucionaria de esa constitución, aunque el producto del proceso sea una nueva constitución, no obstante que el rubro de la Constitución de 1917 exprese que reforma a la Constitución de 1857 se trata solo del discurso no de la realidad.

Con el Constituyente de 1916-1917 y la Constitución que hizo, destruyen la validez de la Constitución de 1857, pero además, debe tomarse en cuenta que si la constitución contiene el querer ser de una nación, la orientación es otra: la de 1857 es individualista y liberal, con un liberalismo social tardío, en la que se monta la constitución de 1917, la primera político social en la historia del constitucionalismo contemporáneo. En su desarrollo, ésta irá socavando la vigencia real de aquélla. No se trata de procesos que quedan plasmados en una fotografía, sino de una película, de un largo y trágico filme.

El paradigma constitucional cambia. De un paradigma de la libertad a un paradigma de la igualdad, lo que resulta una completa novedad, aunque éste deje vigente aquél, pues se trata de un proceso constituyente inclusivo: lo nuevo, pero también lo viejo.

Las ideas de la Constitución de 1917 pertenecían a una minoría, una decisión democrática le hubiera sido desfavorable, por ello afirma Tena Ramírez ${ }^{16}$, que se trata de una constitución impuesta, y efectivamente lo es, impuesta por las armas no por los votos, que poco a poco obtuvo su naturalización a fuerza de sostenerse desde el poder, so pena de sucumbir. Por eso debía imponerse su incorporación en las constituciones de los estados, que tenían poderes no democráticos sino impuestos por las fuerzas carrancistas, conforme al Plan de Guadalupe.

No hay una continuidad constitucional del 1857 a 1917, en sentido estricto, se edifica sobre aquella aprovechando la libertad y utilizándola como discurso para consolidar la nueva constitución, que insisto empezó por imponerse a los estados.

\section{La Recepción de la Constitución Mexicana de 1917 por los Estados de la República}

En esta tesitura de imposición, también a los estados de la república se les impone un constitucionalismo inicialmente inválido, pero que en proceso revolucionario se ve natural, pues el derecho vigente es el que se está gestando para el nuevo querer ser de los mexicanos.

Una situación relevante para el constitucionalismo mexicano se encuentra en dar respuesta a la siguiente cuestión ¿Los estados o entidades federativas son soberanos? La Constitución dice que sí, pero lo son en su régimen interno, aunque todos declaren y se proclamen soberanos, identificando así sus constituciones locales: Constitución Política del Estado Libre y Soberano de. Esta cuestión está estrechamente vinculada con la federación como forma de Estado. Un

\footnotetext{
15 Tena Ramírez, Felipe, op. cit. nota 5, p. 68.

${ }^{16}$ Ibidem. p. 73 .
} 
Estado federal es una unidad en una de sus caras y una pluralidad en la otra, y para mantener la unidad deberán ceder en los aspectos necesarios para ello, definidos por la constitución federal.

Así el régimen interno de los estados es lo que les queda después de cumplir el pacto federal. Entonces, sin estar sometidos jerárquicamente a la Federación, sí están sometidos a la Constitución, lo que incluye a las constituciones locales.

La constitución mexicana de 1917 se expide el 31 de enero, se promulga el 5 de febrero y entra en vigor el $1^{\circ}$ de mayo de 1917, por lo tanto los estados deben armonizar sus constituciones con aquella, estableciendo lo que será su régimen interior.

El 22 de marzo de 1917, Venustiano Carranza, como Primer Jefe del Ejército Constitucionalista y encargado del Poder Ejecutivo de la Unión, con facultades extraordinarias, expide el decreto número 13 para que los estados ajusten sus constituciones a la federal recientemente promulgada.

El decreto se basa en el Plan de Guadalupe y no en la constitución, porque en él Carranza tenía facultades para nombrar y remover libremente gobernadores y comandantes militares en los estados, lo cual resulta entendible y hace posible la armonización pues no iba a poder ordenarles a sus enemigos.

Los gobernadores provisionales de los estados debían convocar a elecciones para elegir a los poderes locales, pero tendrían que ser autorizados por el propio Carranza.

Las legislaturas de los estados que resultaran electas tendrían el carácter de constitucionales y constituyentes limitadas a la armonización constitucional. Sin duda eran constitucionales porque serían parte de la facción constitucionalista, no porque se establecieran conforme a la constitución local; y un constituyente que con los limites naturales es soberano, estaba siendo restringido a una sola tarea, que no era hacer una constitución sino reformarla incorporándole el constitucionalismo de la federal.

\section{El Constitucionalismo en Aguascalientes Producto de la Constitución Mexicana de 1917}

Este proceso y largo hito histórico puede entenderse mejor en la respuesta que damos a esta pregunta: ¿Qué hicieron en Aguascalientes?

La constitución que regía en el Estado de Aguascalientes había sido expedida por el congreso del estado y promulgada por el gobernador, Jesús Gómez Portugal, en el nombre de Dios y con la autoridad del pueblo, en octubre de 1868, como producto de un procedimiento de reforma contenido en la constitución de 1857, la primera del Estado, y para ajustarse a la república restaurada.

La constitución vigente en el Estado de Aguascalientes desde 1868 contemplaba la posibilidad de reforma constitucional en su artículo 117 y el procedimiento establecido en el artículo 118, que por cierto había sido reformado por el General Martín Triana ${ }^{17}$, gobernador y comandante militar, en uso de facultades concedidas y teniendo en consideración lo dispuesto por el Primer Jefe del Ejército Constitucionalista, con fecha 2 de febrero de 1916.

Sin embargo, como triunfantes revolucionarios, por el momento, lo que menos interesaba era respetar la constitución, no obstante su discurso de legalidad, porque para que las adiciones o reformas llegaran a ser parte de la constitución, era menester que se discutieran en el siguiente periodo de sesiones al que fue iniciada, y se requería que el Congreso del Estado las aprobara

\footnotetext{
${ }^{17}$ Muñoz Delgado, José Alfredo (2008), Evolución Constitucional del Estado de Aguascalientes. Ensayo Histórico Constitucional, editorial Epiqueia, México, p. 169.
} 
por el voto de las dos terceras partes de sus integrantes y por el voto de la mayorías de los ayuntamientos del Estado, computando los sufragios individualmente y no por cuerpos.

Otro argumento para demostrar que la constitución federal de 1917 es una nueva constitución es el presupuesto fáctico de que Carranza y su ejército desconocían a las autoridades cuyos cargos se otorgaron conforme a la constitución de 1857. Motivo por el cual ninguna constitución local tenía ya vigencia, y se podían utilizar para montar en ellas la nueva constitución. Así lo decidió Venustiano Carranza en el Decreto número 13, del 22 de marzo de 1917.

En Aguascalientes, los carrancistas desalojan a los huertistas en 1914. El poder era ejercido exclusivamente por el gobernador impuesto por los constitucionalistas. El primero, fue el maderista, Alberto Fuentes Dávila. A fines de año, Aguascalientes, tras la celebración de la Convención Revolucionaria, queda en manos de los villistas quienes, una vez derrotados por Obregón, ceden el poder a los carrancistas en julio de 1915, con Martín Triana, Gregorio Osuna y Antonio Norzagaray, militares incondicionales a Carranza.

Correspondió a Antonio Norzagaray, gobernador constitucionalista desde enero de 1917, dar cumplimiento al Decreto número 13, de fecha 22 de marzo de 1917, convocando a elecciones para Gobernador, Congreso y Magistrados del Supremo Tribunal de Justicia locales.

Con el decreto del 9 de abril de 1917 se convoca a elecciones, las cuales se celebrarían el 13 de mayo siguiente. Para el integrar el congreso del estado serían 15 diputados propietarios y sus respectivos suplentes. En una controvertida elección, de manera abrumadora, es electo Aurelio L. González, un carrancista conservador. Como diputados fueron electos ${ }^{18}$ Mariano Ramos, Manuel S. Flores, Juan Díaz Infante, Rafael Sotura, Blas E. Romo, Manuel I. Ramírez, Jesús Díaz Infante, R. V. Romo, Ezequiel Palacio, Rafael Moran, Juan E. López, Gabriel Landín, Samuel G. García, Alberto E. Pedroza y Samuel J. Guerra.

El periodo para el cual fueron electos empezaba el 16 de septiembre de 1916 y duraría lo que la constitución determinara. La constitución de 1868 determinaba en su artículo 53 que la Legislatura se renovaría en su totalidad cada dos años, periodo que coincide con la Constitución de 1917, en su artículo 15. Sin embargo, empezaron el ejercicio de su función específica en primer término; como constituyentes, armonizarían la Constitución local de 1868 con la constitución federal de 1917 teniendo como límite para ello el 16 de septiembre de 1917, debido a que a partir de esa fecha empezarían su ejercicio como poder legislativo ordinario.

El 3 de septiembre de 1917 se terminan los trabajos de armonización y el día 6 se expide la Constitución, la cual se publica en El Republicano, periódico oficial del gobierno del estado, el día 9 del mismo mes y año.

Se instituyeron un congreso constituyente federal como otros tantos locales. Así convenía a la facción triunfante en ese momento de nuestra revolución, como una acción de fuerza realizada no en los campos de batalla sino en el campo ideológico. Podría decirse que fue otra revolución sin balas ni sangre, una revolución de ideas y formas jurídicas que se abrieron paso en el devenir histórico del siglo XX mexicano.

\section{Reflexión Final}

Queda concluir lo que Vera Estañol afirma:

\footnotetext{
${ }^{18}$ LII Legislatura, Congreso del Estado de Aguascalientes (1986), Las Constituciones de Aguascalientes, México, p. 107.
} 
La Constitución de 1917 es ilegítima, también las reformas a la Constitución de Aguascalientes de 1868, porque las asambleas constituyentes, tanto de Querétaro, como la de Aguascalientes, no representaban la voluntad popular sino a la facción armada más numerosa, en el momento en que se expiden los textos fundamentales, la minoría de carrancistas o constitucionalistas como paradójicamente se hacían llamar ${ }^{19}$.

Hubieran reivindicado el derecho a la revolución, al tiranicidio incluso, no la constitución de 1857.

Aunque a la postre también Carranza sería devorado por la Revolución, dejaba el legado constitucional que no acertó a cambiar el grupo sonorense que terminó alzándose con el triunfo e institucionalizándose en el poder.

En México, la simulación constitucional ha sido la nota definitoria de nuestro derecho público, es una máscara, útil para mirar al mundo. Y aunque esto sea un secreto a voces, parece que hay un pacto en el sustrato social para no revelarlo, nos sentimos cómodos con ella y preocupados por conservarla.

\section{Fuentes bibliográficas}

DíAz y DíAz, Martín (1991), Emilio Rabasa teórico de la dictadura necesaria, Miguel Ángel Porrúa, México.

Gobierno Provisional de la República Mexicana (1915) Codificación de Decretos del C. Venustiano Carranza, Primer Jefe del Ejército Constitucionalista, encargado del Poder Ejecutivo de la Unión, Imprenta de la Secretaria de Gobernación, México.

Iglesi As, José María (1874), Estudio Constitucional Sobre las Facultades de la Corte Suprema, Imprenta de Díaz de León y White, México.

Krauze, Enrique (1987), Venustiano Carranza. Biografía del Poder 5, Fondo de Cultura Económica, México.

LII Legislatura, Congreso del Estado de Aguascalientes (1986), Las Constituciones de Aguascalientes, México.

Monsiváis, Carlos (1986), Prólogo, a La Bola de Emilio Rabasa. Ediciones Océano, México.

Muñoz Delgado, José Alfredo (2008), Evolución Constitucional del Estado de Aguascalientes. Ensayo Histórico Constitucional. Editorial Epiqueia, México.

Rabasa, Emilio (1912), La Constitución y la Dictadura, Tipografía de Revista de Revistas, México.

Solares, Ignacio (2013), Un sueño de Bernardo Reyes, Alfaguara, México. (2009), Ficciones de la Revolución Mexicana. Alfaguara, México.

Tena Ramírez, Felipe (1981), Derecho Constitucional Mexicano. Editorial Porrúa. México.

\footnotetext{
${ }^{19}$ Vera Estañol, Jorge (1919), Al Margen de la Constitución de 1917, Wayside Prees, Los Ángeles, Cal. EE.UU. 
(1957), Leyes Fundamentales de México, Porrúa, México.

VAsconcelos, José (2009), ¿Qué es la Revolución?, Editorial Trillas, Biblioteca José Vasconcelos No. 7, México.

Vera Estañol, Jorge (1919), Al Margen de la Constitución de 1917, Wayside Prees, Los Ángeles, Cal. EE.UU. 
